\title{
FAR-ULTRAVIOLET NUMBER COUNTS OF FIELD GALAXIES
}

\author{
Elysse N. Voyer ${ }^{1,5}$, Jonathan P. Gardner ${ }^{2}$, Harry I. TePlitz ${ }^{3}$, Brian D. Siana ${ }^{4}$, and Duilia F. De Mello ${ }^{1,2}$ \\ ${ }^{1}$ Department of Physics, The Catholic University of America, Washington, DC 20064, USA; 48voyer@ cardinalmail.cua.edu \\ ${ }^{2}$ Astrophysics Science Division, Observational Cosmology Laboratory, Goddard Space Flight Center, Code 665, Greenbelt, MD 20771, USA \\ ${ }^{3}$ Spitzer Science Center, California Institute of Technology, 220-6, Pasadena, CA 91125, USA \\ ${ }^{4}$ California Institute of Technology, MS 105-24, Pasadena, CA 91125, USA \\ Received 2010 July 14; accepted 2011 May 6; published 2011 July 11
}

\begin{abstract}
The far-ultraviolet (FUV) number counts of galaxies constrain the evolution of the star formation rate density of the universe. We report the FUV number counts computed from FUV imaging of several fields including the Hubble Ultra Deep Field, the Hubble Deep Field North, and small areas within the GOODS-North and South fields. These data were obtained with the Hubble Space Telescope (HST) Solar Blind Channel of the Advance Camera for Surveys. The number counts sample an FUV AB magnitude range from 21 to 29 and cover a total area of $15.9 \operatorname{arcmin}^{2}, \sim 4$ times larger than the most recent HST FUV study. Our FUV counts intersect bright FUV Galaxy Evolution Explorer counts at $22.5 \mathrm{mag}$ and they show good agreement with recent semi-analytic models based on dark matter "merger trees" by R. S. Somerville et al. We show that the number counts are $\sim 35 \%$ lower than in previous HST studies that use smaller areas. The differences between these studies are likely the result of cosmic variance; our new data cover more lines of sight and more area than previous HST FUV studies. The integrated light from field galaxies is found to contribute between $65.9_{-8}^{+8}$ and $82.6_{-12}^{+12}$ photons s $\mathrm{cm}^{-2} \mathrm{sr}^{-1} \AA^{-1}$ to the FUV extragalactic background. These measurements set a lower limit for the total FUV background light.
\end{abstract}

Key words: cosmology: observations - galaxies: evolution - galaxies: statistics - ultraviolet: galaxies

Online-only material: color figures

\section{INTRODUCTION}

Measuring the number counts of field galaxies within an observed area as a function of magnitude is one of the fundamental techniques used to study galaxy evolution throughout cosmic time. Galaxy number counts are used to test theoretical models of galaxy evolution; changes in the slope of number count distributions reflect physical changes in the underlying galaxy populations. Such models can predict galaxy properties in various bandpasses and for various redshifts $(z)$. At observed farultraviolet (FUV) wavelengths, galaxy counts probe light from unobscured star formation for $z<1$, after which the Lyman limit (912 $\AA$ ) shifts into the observed bandpass. Little to no UV light is detectable blueward of this limit because it is used in ionizing $\mathrm{H}_{\mathrm{I}}$ gas in the interstellar and intergalactic medium (IGM) between the galaxy and the observer. This has been shown in several studies attempting to constrain the Lyman continuum escape fraction at various redshifts (i.e., Siana et al. 2010, 2007; Bridge et al. 2010; Cowie et al. 2009). The majority of the detected UV light is radiated by hot, massive, $\mathrm{O}$ and $\mathrm{B}$ stars that have spectral energy distributions (SEDs) peaking at these wavelengths. Due to their short lifetimes, the UV light from $\mathrm{O}$ and B stars traces the star-forming regions within galaxies. For this reason, the number counts of UV detected galaxies provide a window into ongoing extragalactic star formation history.

FUV number count studies are only possible with spacebased observations since Earth's atmosphere is opaque to UV light. Over the past two decades, only a handful of spacebased field galaxy surveys have been carried out at UV wavelengths (Milliard et al. 1992; Deharveng et al. 1994; Gardner et al. 2000a; Iglesias-Páramo et al. 2004; Xu et al. 2005; Teplitz et al. 2006; Hoversten et al. 2009) since long integration times are required to reach faint magnitudes. The first UV

\footnotetext{
5 NASA Graduate Student Research Program Fellow.
}

galaxy counts were measured by Milliard et al. (1992) using the balloon-borne FOCA instrument at $2000 \AA$ and bright magnitudes 15-18.5, covering a large area of sky $\left(\sim 6 \mathrm{deg}^{2}\right)$. Later, two studies used deep imaging from the Hubble Space Telescope (HST) to measure faint UV galaxy counts. Gardner et al. (2000a) measured NUV (2365 $\mathrm{A})$ and FUV (1595 $\mathrm{A})$ counts over smaller areas (1.54 $\left.\operatorname{arcmin}^{2}\right)$ for magnitudes 24.5-29.5 in the Hubble Deep Fields-North and South (HDF-N and S). Teplitz et al. (2006) measured FUV (1600 $)$ counts for magnitudes 20.5-28.5 in the HDF-N, covering $3.77 \mathrm{arcmin}^{2}$. Bright UV galaxy counts (NUV: 2310, FUV: 1530), between 14 and $23.8 \mathrm{mag}$, were measured by Xu et al. (2005) using 36 Mediumdepth Survey fields and 3 Deep Survey fields obtained with the Galaxy Evolution Explorer (GALEX). They cover a total area $\sim 20 \mathrm{deg}^{2}$. More recently, Hoversten et al. (2009) used the Swift UV/Optical Telescope to measure NUV (1928 A, $2246 \AA, 2600 \AA$ ) galaxy counts in a $289 \operatorname{arcmin}^{2}$ area of the Chandra Deep Field-South (CDF-S) between 21 and 26 mag. However, the only two studies measuring the faint-end slope (24.5-29.5 mag) of the FUV galaxy counts are subject to cosmic variance effects, due to the small areas surveyed, and known overdensities in the HDF-N (Cohen et al. 2000).

In this paper, we present FUV (1614 $\AA$ ) galaxy number counts from deep images obtained with HST's Solar Blind Channel (SBC) on the Advanced Camera for Surveys (ACS). These observations sample a magnitude range of 21.5-29.5 and cover an area $\sim 4$ times larger $\left(15.9 \operatorname{arcmin}^{2}\right)$ than the most recent FUV study that previously covered the largest area at these wavelengths and magnitudes (Teplitz et al. 2006). In Section 2, we present the data used for this study. In Section 3.1, we discuss the measurement of the number counts and corrections to the counts due to observational biases. The number counts are compared with previous studies in Section 3.2 and theoretical models in Section 3.3. Cosmic variance is discussed in Section 3.4, and the FUV extragalactic background 


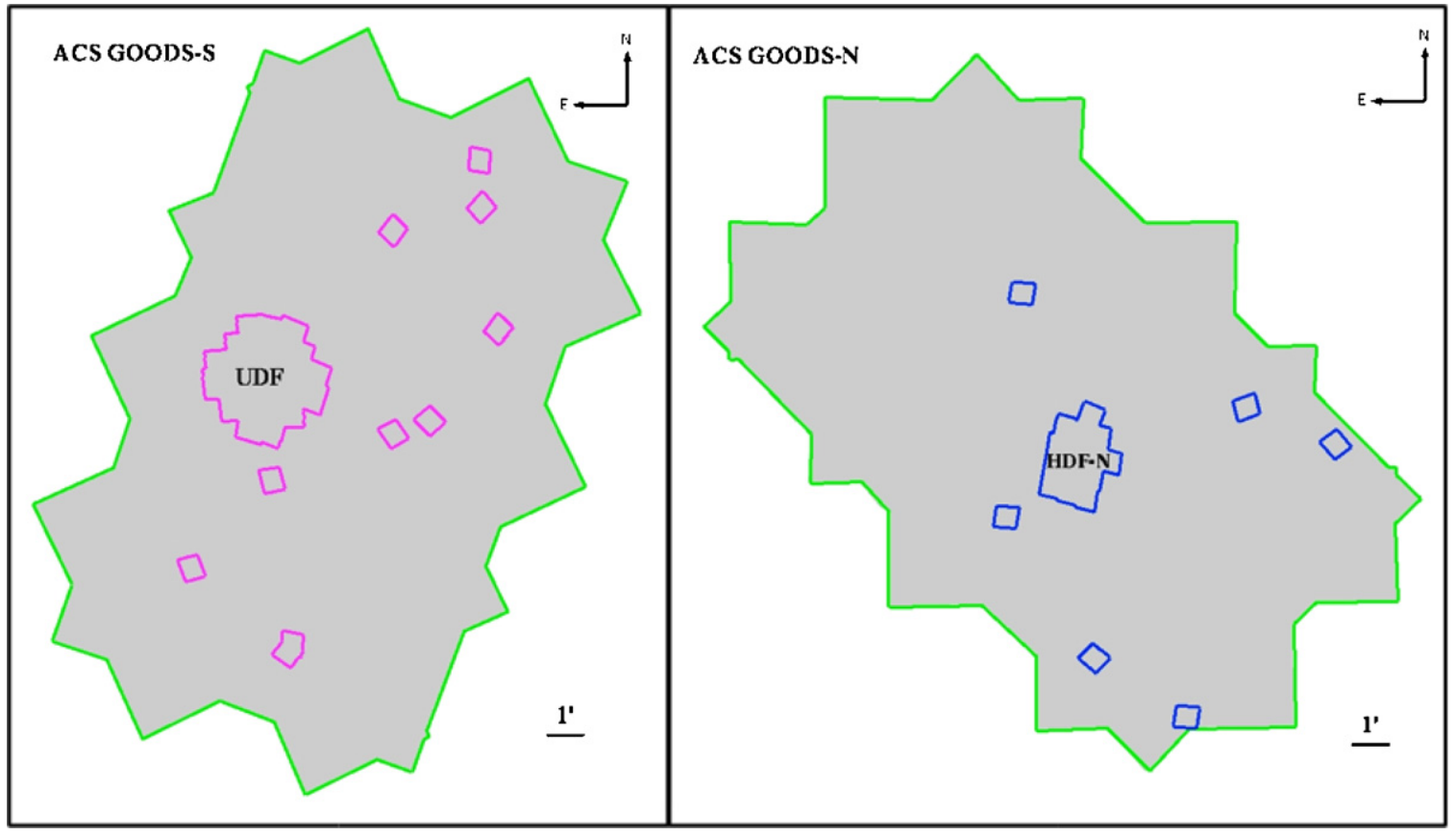

Figure 1. Footprints of regions observed with the ACS SBC within the ACS GOODS-N and S areas.

(A color version of this figure is available in the online journal.)

light (EBL) calculation is presented in Section 3.5. Results of this study are summarized in Section 4.

\section{THE DATA}

For this study, we used FUV observations from three different data sets: the HDF-N area of the Great Observatories Origins Deep Survey North (GOODS-N) field, the Hubble Ultra Deep Field (HUDF) area of the GOODS-South (GOODS-S) field, and smaller fields in various parts of the GOODS-N and S fields (see Figure 1). The HDF-N data are from the HST General Observer Program 9478, the HUDF data are from the HST Cycle 13 Treasury Program 10403, and the smaller GOODS-N and S fields are from the HST Cycle 15 General Observer Program 10872. All observations were obtained with the SBC detector on Hubble's ACS. The ACS SBC detector is a Multi-Anode Microchannel Array with a field of view of $344^{\prime \prime} 6 \times 30^{\prime \prime} .8$. All observations were taken through the long-pass quartz filter, F150LP, that peaks $\sim 1500 \AA$, has a bandwidth of $\sim 550 \AA$, effective wavelength of $1614 \AA$, and an FWHM $=177 \AA$. At $z \sim 0.6$ the Lyman limit, $912 \AA$, is bandshifted to $1500 \AA$, thus the SBC F150LP is only sensitive to the brighter galaxies beyond $z>0.7$.

Final images of the HDF-N and HUDF used for source detection were constructed using the DRIZZLE package in IRAF. $^{6}$ The smaller GOODS-N and S images were tiled onto the original GOODS areas for source detection. Photometry is performed using similar procedures to those in Gardner et al. (2000b) and Teplitz et al. (2006) where optical segmentation maps produced with the SExtractor software package (Bertin \& Arnouts 1996) are used to determine the pixels that are included in the FUV flux measurement. SExtractor has difficulty working on data with few counts per pixel (Gardner et al. 2000b), such as the FUV images, thus in order to prevent false segmentation of

6 IRAF is distributed by the National Optical Astronomy Observatory, which is operated by the Association of Universities for Research in Astronomy, Inc., under cooperative agreement with the National Science Foundation. sources, the optical image is used for detection because galaxy morphologies are less clumpy in the rest-frame optical than in the UV (Teplitz et al. 2006). We used SExtractor to detect sources in the GOODS-N and S fields ACS F606W ( $V$-band) images and defined extraction isophotes that extend out to where the galaxy flux per pixel is 0.8 times the background root mean square $(\mathrm{rms} ; \sigma)$. The $0.8 \sigma$ apertures are then used to extract fluxes in the FUV images. The F606W images (Beckwith et al. 2006; Giavalisco et al. 2004) are more than 1 mag deeper than the FUV images (in AB mags). Thus, we can be confident that we are capturing all of the FUV flux within these apertures.

Galactic extinction does not vary significantly over the areas we observe because the GOODS fields were selected in part for the low extinction along their sight lines (Beckwith et al. 2006; Williams et al. 1996). From the Galactic dust maps of Schlegel et al. (1998), ${ }^{7}$ we find the range of extinction to be small over the GOODS-N and S fields, varying between $0.0347 \leqslant A_{V} \leqslant$ 0.0381 and $0.0236 \leqslant A_{V} \leqslant 0.0298$, respectively. From these dust maps, we find $A_{V}$ at the central coordinate of each FUV source and calculate the corresponding amount of extinction in the FUV, $A_{1610}$, via the ratio given in Siana et al. (2010) based on the extinction curve of Cardelli et al. (1989): $A_{1610} / A_{V}=$ 2.55. None of the corrections for Galactic extinction are larger than $10 \%$.

We detected 114 FUV sources in the HUDF area of GOODS-S, 113 FUV sources in the smaller GOODS-N and $\mathrm{S}$ images, and $116 \mathrm{FUV}$ sources from the HDF-N area of GOODS-N. We removed 10 sources because they were too close to the edges of the images, leaving 333 sources to be included in the measurement of the number counts. Three sources are also Chandra X-ray detections CXO J123648.0+621309, CXO J033239.0-274602, and CXO J333213.2-274241 (Evans et al. 2010) located in the HDF-N area of GOODS-N, the UDF area of GOODS-S, and a smaller area of GOODS-S, respectively. No

\footnotetext{
7 Accessed via the NASA/IPAC Infrared Science Archive (IRSA) Galactic Dust Extinction tool.
} 


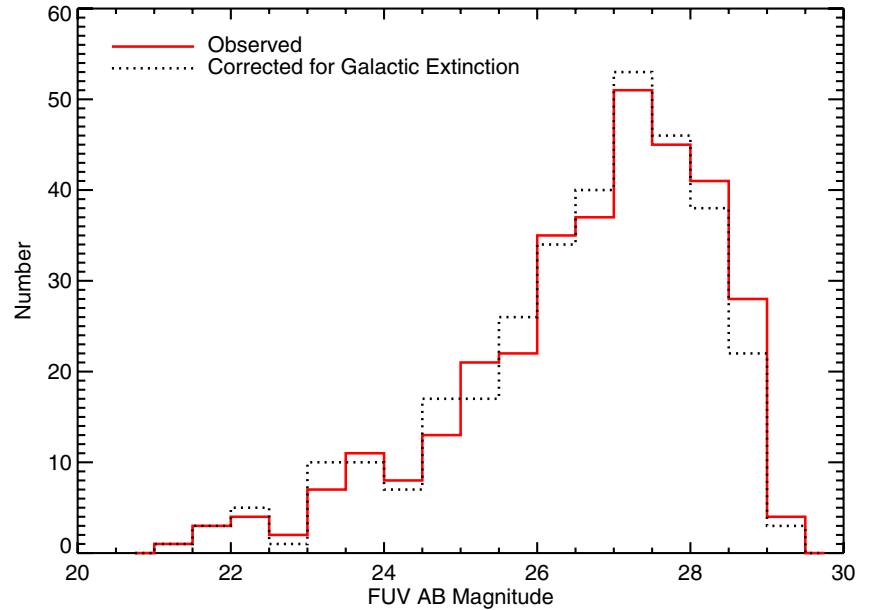

Figure 2. FUV magnitude distribution for the 333 sources included in FUV number counts. Both the magnitude distribution as observed and the magnitude distribution corrected for Galactic extinction are shown. The extinction correction was done with $A_{V}$ values from the Schlegel et al. (1998) Galactic dust maps and the ratio of $A_{1610} / A_{V}=2.55$ calculated in Siana et al. (2010).

(A color version of this figure is available in the online journal.)

stars were detected in our sample. The total sample covers an AB magnitude range from 21 to 29 and its magnitude distribution begins to drop-off at $\sim 28.5$ as shown in Figure 2. Redshifts are available for 212 sources (Dahlen et al. 2010; Cohen et al. 2000; T. Dahlen 2004, private communication) and the distribution for the FUV sample is shown in Figure 3.

\section{NUMBER COUNTS IN THE FUV}

\subsection{Measurement of Number Counts}

In order to measure the number counts of galaxies in our sample, we used the method developed in Gardner et al. (2000a). Because there are variations in depth across the FUV images, each FUV source would not necessarily be detectable over an entire image. For each source we must calculate the total area in which it would have been detected in each image. We use the rms error maps, produced from the weight maps of the drizzled SBC images, to determine these areas. Small-scale variations created during the image drizzling process are accounted for by smoothing the rms maps with a $0.4 \times 0$ '. 4 median filter. $\mathrm{AB}$ magnitude and size of each source are required to calculate the total detection area in an image for that source. FUV AB magnitudes $\left(\mathrm{FUV}_{\mathrm{AB}}\right)$ of the sources in all observed fields were obtained from photometric catalogs produced with SExtractor as described in Section 2. The size of each source was determined from the SExtractor segmentation maps used for the catalog photometry. Using the size and magnitude of each source, we calculate the maximum rms error of a pixel at $3 \sigma$ in the following way: flux $/(3 \times \sqrt{\text { size }})$. Pixels in the rms map with errors less than or equal to this value make up the total area over which the source would have been detected at $3 \sigma$.

In order to be consistent, and not overestimate the detection area of the other sources, we cut down the edges of each rms map by a length equal to the radius of the circular area of each removed source (discussed in Section 2) before calculating their detection areas. This includes both the outer edges of images as well as edges on the inner parts of the images where drizzled fields do not overlap in the HUDF and HDF-N. In any given magnitude bin, there might be a failure to detect low surface brightness objects that are actually there. This effect can

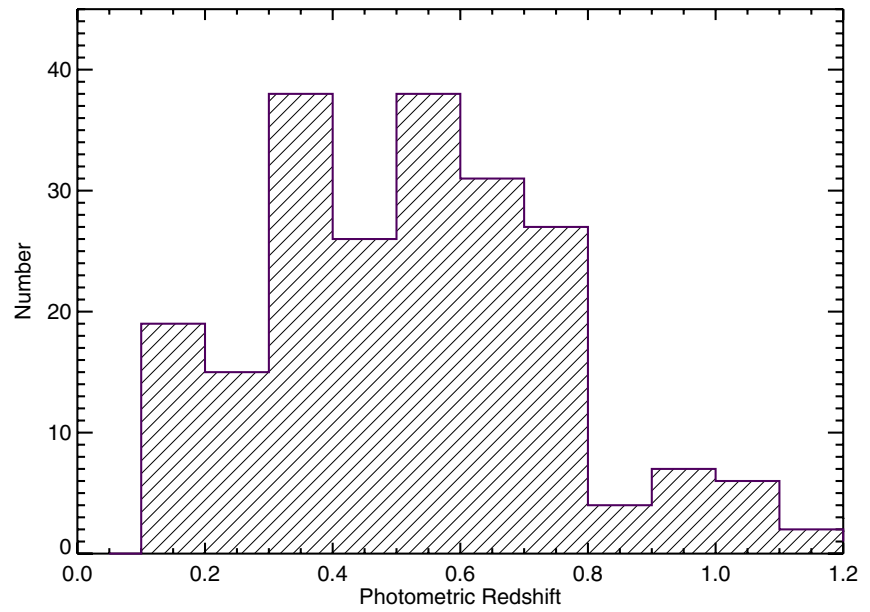

Figure 3. Distribution of photometric redshifts, where available, for 212 sources from the FUV number counts sample (Dahlen et al. 2010; T. Dahlen 2004, private communication; Cohen et al. 2000).

(A color version of this figure is available in the online journal.)

become larger toward fainter magnitudes. Thus, number count measurements must be adjusted for such incompleteness biases. To correct for incompleteness, we use two independent methods, bootstrap-sampling, and detection of artificially introduced galaxies. For the first method we bootstrap-sample the size distribution of the version 2.0 GOODS-S $V$-band catalog 8 starting with a randomly generated FUV galaxy sample. First, 1000 FUV magnitudes are randomly generated for each FUV magnitude bin. Next, following the procedure in Gardner et al. (2000a), we use the mean and standard deviation parameters of a Gaussian distribution fit to the FUV $-V$ color distribution in the HUDF between $24 \leqslant m_{\mathrm{AB}} \leqslant 28$ to randomly generate FUV $-V$ colors. Even though the completeness correction is ultimately applied to all magnitude bins from 21.5 to 29.5 , this range $\left(24 \leqslant m_{\mathrm{AB}} \leqslant 28\right)$ is selected for determining the Gaussian distribution because the magnitude distribution of the FUV sample drops off at $\sim 28.5$, and there are very few galaxies in the HUDF with magnitudes brighter than 24 . Thus, including bins brighter or fainter than these magnitudes would introduce unwanted errors into the distribution. Because the Gaussian color distribution does not vary greatly between the different SBC fields we only sample the HUDF. With these random FUV magnitudes and FUV- $V$ colors, we calculate the optical magnitudes of the randomly generated sample and match them to the closest optical magnitudes of sources in the GOODS-S $V$-band catalog. The sizes of these objects are then sampled from segmentation maps produced from public GOODS-S $V$-band images with SExtractor using $0.8 \sigma$ isophotes to define the source areas. These are isophotes within which each pixel in the $V$-band images is $0.8 \sigma$ above the background noise. We use a $0.8 \sigma$ size isophote because the same is used to define the source areas for the FUV photometry. From the sizes and FUV magnitudes of the random sample, we then calculate the maximum rms pixel error below which each simulated object would be detected and proceed to calculate the total detection area for each simulated object in the SBC rms maps. Finally, to get the completeness correction factor for each magnitude bin, we average per magnitude bin the detection areas of the simulated objects (including galaxies with zero detection area)

\footnotetext{
8 http://archive.stsci.edu/prepds/goods/
} 
Table 1

FUV Galaxy Counts

\begin{tabular}{|c|c|c|c|c|c|c|c|}
\hline $\begin{array}{l}\mathrm{FUV}_{\mathrm{AB}} \\
(\mathrm{mag})\end{array}$ & $\begin{array}{c}\mathrm{NC} \\
\left(\text { No. } \mathrm{deg}^{-2} \mathrm{mag}^{-1}\right)\end{array}$ & $\log \mathrm{NC}$ & $\sigma_{\text {low }}$ & $\sigma_{\text {high }}$ & Raw No. & Completeness & $\begin{array}{c}\text { Area } \\
\left(\operatorname{arcmin}^{2}\right)\end{array}$ \\
\hline 21.5 & 937 & 2.97 & 0.26 & 0.27 & 4 & 1.054 & 13.89 \\
\hline 22.5 & 1402 & 3.15 & 0.20 & 0.23 & 6 & 1.056 & 13.89 \\
\hline 23.5 & 4656 & 3.67 & 0.10 & 0.12 & 20 & 1.026 & 14.70 \\
\hline 24.5 & 5582 & 3.75 & 0.09 & 0.11 & 24 & 1.019 & 14.91 \\
\hline 25.5 & 9996 & 4.00 & 0.07 & 0.07 & 43 & 1.007 & 15.28 \\
\hline 26.5 & 17207 & 4.24 & 0.08 & 0.03 & 74 & 1.003 & 15.42 \\
\hline 27.5 & 25166 & 4.40 & 0.04 & 0.05 & 99 & 0.972 & 15.20 \\
\hline 28.5 & 18752 & 4.27 & 0.06 & 0.06 & 60 & 0.854 & 14.33 \\
\hline 29.5 & 2041 & 3.31 & 0.28 & 0.36 & 3 & 0.317 & 14.56 \\
\hline
\end{tabular}

Notes. Magnitudes represent the center of the bins, errors are 1 $\sigma$ Poissonian (Gehrels 1986), and areas are the average total detection areas of all objects within each magnitude bin.

and take the ratio with the average detection area of all real FUV sources in corresponding bins.

The second incompleteness correction method introduces 500 artificial FUV galaxies into the SBC data for each magnitude bin and recovers them with the same photometry algorithm used for the real data. We simulate these sources using the IRAF task ARTDATA in the NOAO package. We also simulate $500 \mathrm{~V}$-band galaxies using the same software in order to use their isophotal sizes to provide the area in which to measure the flux of the artificial FUV sources. This approach mimics the procedure of the actual FUV photometry. To determine the correct magnitudes of the artificial $V$-band galaxies, we use the parameters of the same Gaussian FUV- $V$ color distribution discussed above. An FUV artificial source that has sinal-tonoise ratio $>3.5$ is a detection, and the detection ratio equals the number of sources recovered over 500. Finally, to correct for incompleteness the number counts are divided by the detection ratio in each magnitude bin.

Both methods yield similar incompleteness corrections, within a few percent of one another, in each bin. An average of these two methods is used for the final correction to the number counts.

\subsection{Comparison with Previous FUV Number Counts}

In Figure 4, we present the completeness corrected and the raw FUV number counts from this work and past FUV number counts from the literature. Their measured values, errors, completeness, and detection areas per magnitude bin are provided in Table 1. Small number Poisson statistical errors are calculated for each point from Gehrels (1986) at the $1 \sigma$ level. The filled circles represent our completeness corrected counts. The open circles represent the raw counts. The upside-down triangles represent counts done with SBC images of the HDF-N from Teplitz et al. (2006). The asterisks represent counts done with HST STIS in the HDF-N and HDF-S from Gardner et al. (2000a). The squares represent counts done with GALEX from $\mathrm{Xu}$ et al. (2005, hereafter XU05 fields), and the upright triangles represent counts done with GALEX from Hammer et al. (2010, hereafter HAM10 field). No color corrections are made between the SBC filter which has a central wavelength of $1614 \AA$ (filter peak is $\lambda=1500 \AA$ ) and the STIS and GALEX filters that have FUV central wavelengths at $1595 \AA$ and $1530 \AA$, respectively. As discussed in Teplitz et al. (2006), the color correction between the SBC and GALEX FUV filters would be significant for galaxies at $z>0.50$ because the SBC filter is sensitive to a larger volume $(\sim 30 \%)$ than the GALEX filter. This color

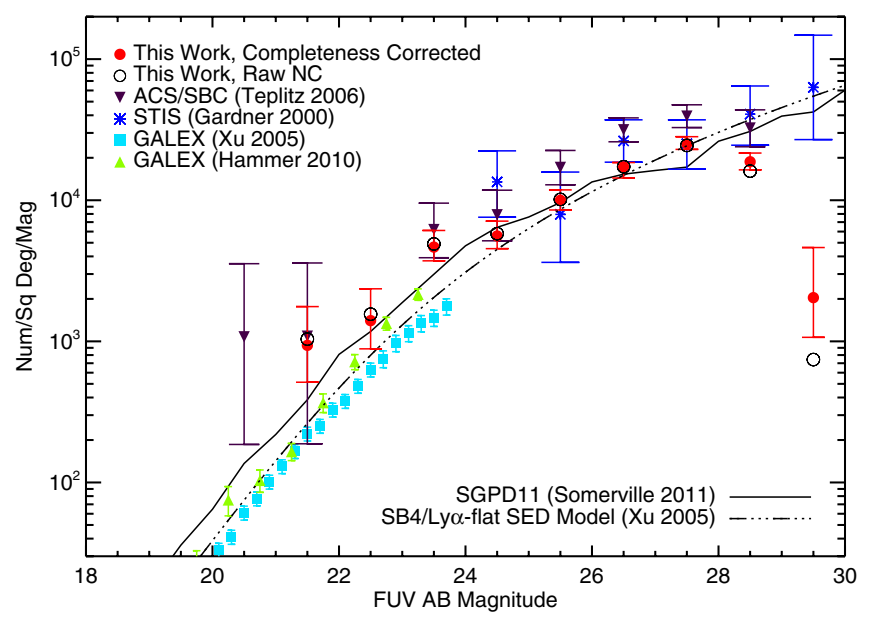

Figure 4. FUV number counts of field galaxies from this work shown with FUV number counts from previous studies and compared to semi-analytic models. Error bars are Poissonian from Gehrels (1986). The caps of the error bars do not reflect an error in magnitude, but have been manually varied in length to better distinguish among them.

(A color version of this figure is available in the online journal.)

difference results in no more than a factor of $\sim 2(\sim$ half a magnitude) between the SBC and GALEX number counts. They also discuss that Ly $\alpha$ emitting sources at $z<0.15$ could have the opposite effect resulting from the bluer wavelength coverage of the GALEX filter. About $54 \%$ of our FUV sample with $z_{\text {phot }}$ are at $z_{\text {phot }}>0.50$ and $\sim 2.3 \%$ are at $z_{\text {phot }}<0.15$. The majority $(98 \%)$ of sources at $z_{\text {phot }}>0.5$ are not comparable to GALEX bins because they have fainter magnitudes ( $\left.\mathrm{FUV}_{\mathrm{AB}}>24\right)$. Thus, comparisons with GALEX FUV number counts are not largely affected by ignoring the filter color correction.

Our galaxy sample probes the faint end of the FUV number counts, with the majority of sources occupying magnitude bins 23.5-28.5. This is reflected in the error bars of these plotted points. The three faintest objects in our sample have $\mathrm{FUV}_{\mathrm{AB}}=$ 29.19, 29.21, and 29.33. Although these sources are fainter than the magnitude drop-off of the FUV data $(\sim 28.5)$, they are detected in the GOODS $V$-band catalog and above the detection threshold of $0.8 \sigma$. Thus, they are included in these number counts. However, due to the few sources detected, the measurement does not accurately represent the number counts at this faint level. On average our number counts are $\sim 35 \%$ and $\sim 36 \%$ lower than the faint HST FUV counts from Gardner et al. (2000a) and Teplitz et al. (2006), respectively. The differences 
in the measurements are likely the result of cosmic variance which is discussed in more detail in Section 3.4.

At the $22.5 \mathrm{mag}$ bin, the slope of our number counts intersects the faint end of the GALEX HAM10 field counts but not the XU05 field counts, remaining higher than these at all overlapping magnitudes. It is not well understood why the GALEX counts diverge from each other after $\mathrm{FUV}_{\mathrm{AB}} \sim 21.25$, but Hammer et al. (2010) show that the divergence cannot be due to their source detection/photometry methods, active galactic nuclei (AGNs), or cosmic variance between fields. Also, while cluster members in the HAM10 field bias the bright bins of these number counts, they only compose $\sim 2 \%$ of objects in the faintest bin, which represents the limiting depth of the survey. However, massive clusters are known to be associated with many filaments and the number of filaments is directly correlated with cluster mass (Pimbblet et al. 2004). Thus, Hammer et al. (2010) do not rule out large-scale structure behind the massive Coma Cluster as the culprit of their excess galaxy counts.

\subsection{Comparison with Number Count Models}

A primary use of galaxy number counts is to test and constrain models of galaxy evolution. In Figure 4, we compare our FUV number counts with two different models, a simple luminosity evolution model from $\mathrm{Xu}$ et al. (2005) and a cosmological semi-analytic model (SAM) from Somerville et al. (2011, hereafter SGPD11). The first model is the SB4/Ly $\alpha$-flat SED model. This model is characterized by a UV luminosity evolution, $L^{*} \sim(1+z)^{2.5}$, and is constructed from a local FUV luminosity function (Wyder et al. 2005) with an estimated $K$-correction based on the UV SB4 SED from Kinney et al. (1996) with a flat spectrum between $1200 \AA$ and $1000 \AA$. It was selected as an initial check that our measured number counts were reasonable since this model is in good agreement with evolution models derived from observed luminosity functions at high- $z$ (Arnouts et al. 2005). When plotting the SB4/Ly $\alpha$ flat SED model we did not color correct the model from the GALEX FUV effective wavelength at $1530 \AA$ to the SBC effective wavelength at $1614 \AA$ (see the further discussion in Section 3.2).

The second model, SGPD11, makes use of the latest version of the SAMs developed by Somerville and collaborators (Somerville et al. 2008, 2001; Somerville \& Primack 1999). The backbone of these SAMs is dark matter "merger trees" representing the hierarchical build-up of structure in the $\Lambda$ cold dark matter $(\Lambda \mathrm{CDM})$ paradigm. The model shown here is the "fiducial WMAP5" model presented in SGPD11 and adopts cosmological parameters consistent with the five-year Wilkinson Microwave Anisotropy Probe (WMAP) analysis (WMAP5; $\left.\Omega_{m}=0.2383, \Omega_{\Lambda}=0.7617, h=0.732, \sigma_{8}=0.82\right)$. The physical processes included in the model include radiative cooling of gas, photoionization squelching, star formation in quiescent and burst modes, morphological transformation via mergers, supernovae feedback, chemical evolution, black hole growth, AGN-driven winds, and radio-mode feedback. The UV luminosities for the SAM galaxies are calculated from synthetic SEDs created by convolving the star formation and chemical enrichment histories for each galaxy with Bruzual \& Charlot (2003) stellar population models using a Chabrier initial mass function. A two-component model for extinction by dust in diffuse cirrus and in dense "birth clouds," following Charlot \& Fall (2000), is also applied (for details see SGPD11). SGPD11 found, in agreement with other studies, that they had to adopt dust parameters that varied with redshift in order to match the
UV and $B$-band luminosity functions at high redshift. We note that unlike simple pure luminosity evolution models, SAMs have many physical sources of scatter in galaxy number densities and properties.

We compare our number counts to the SGPD11 model for several reasons. This model includes what are believed to be the key physical processes that shape galaxy formation and evolution. In particular, the FUV number counts are expected to provide an important constraint on the processes that trigger and regulate star formation, which are highly uncertain. The FUV number counts are also highly sensitive to dust extinction, which is another uncertain ingredient in the SAMs.

Our measured FUV number counts are broadly consistent with the SGPD11 and the SB4/Ly $\alpha$-flat SED model over all magnitudes. As seen in Figure 4, the SB4/Ly $\alpha$-flat SED model appears lower than the SGPD11 SAM up to $\mathrm{FUV}_{\mathrm{AB}} \sim 26.5$ after which the trend is reversed and the SGPD11 model is lower. The differences in the bright end of the models are most likely due to the fact that the SB4/Ly $\alpha$-flat SED model is derived from a single SED. Both sets of GALEX counts are lower than the SGPD11 model at the bright end, however the HAM10 field counts start to coincide with the models at $\mathrm{FUV}_{\mathrm{AB}}>$ 22.5. This is consistent with the fact that the SGPD11 model is known to overproduce bright galaxies compared to GALEX data (Gilmore et al. 2009; Somerville et al. 2011), due to a small degree of residual "overcooling" in massive halos. Our number counts do not match the SB4/Ly $\alpha$-flat SED model at all magnitudes, but begin to coincide with it after $24.5 \mathrm{mag}$. As discussed by Teplitz et al. (2006), the discrepancies with this model, especially toward bright magnitudes, may suggest a need for number density evolution in FUV galaxy number count models because this model only takes into account luminosity evolution.

\subsection{Effects of Cosmic Variance}

Uncertainties in measurements of galaxy number counts can arise as a result of overall large-scale structure variation or cosmic variance (Somerville et al. 2004). The observations used for this study were designed to significantly reduce the effects of cosmic variance by including data from various sight lines and covering a larger area than any previous FUV number counts study at these wavelengths and magnitudes. Our observations cover a total area of $15.9 \operatorname{arcmin}^{2}$, while the Gardner et al. (2000a) STIS observations in the HDF-N and S cover only $1.54 \operatorname{arcmin}^{2}$ and the Teplitz et al. (2006) SBC observations in the HDF-N cover only $\sim 3.77 \operatorname{arcmin}^{2}$. Also, the HDF-N has galaxy overdensities at $z \sim 0.45$ and $z \sim 0.8$ (Cohen et al. 2000) that bias the number counts in that field. To demonstrate the effects of cosmic variance, we have compared in Figure 5 our total FUV number counts with the number counts calculated in the GOODS-N and GOODS-S SBC fields separately. The red circles represent the total number counts, blue upside-down triangles represent the number counts in the GOODS-S area, and the orange squares represent the number counts in the GOODS$\mathrm{N}$ area. The counts in the GOODS-N area are consistently higher than those in GOODS-S in every magnitude, bin except 22.5. The total number counts are a clear average of the number counts in these two fields over the entire magnitude range. This result demonstrates that using large areas and various sight lines to make measurements of number counts reduces bias due to cosmic variance, and ideally these types of data sets provide the best comparisons for SAMs. 


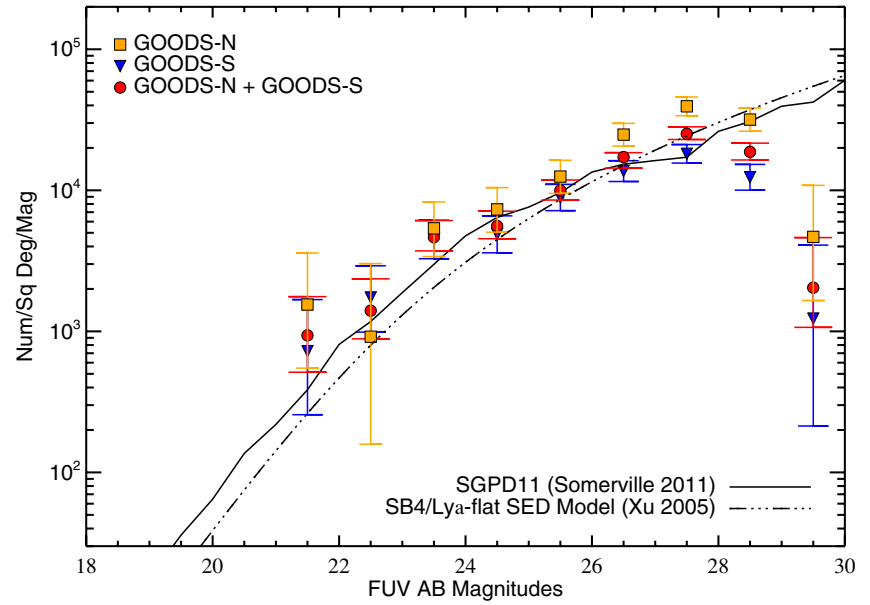

Figure 5. FUV number counts for individual fields. We excluded the brightest (21.5 mag) and the faintest (29.5 mag) magnitude bins from this plot because there is not enough signal-to-noise to make a comparison between fields at these magnitudes. HDF-N counts are from Teplitz et al. (2006). The caps of the error bars do not reflect an error in magnitude, but have been manually varied in length to better distinguish among them.

(A color version of this figure is available in the online journal.)

\subsection{The FUV Background Light from Resolved Sources}

The total UV background light is composed of several ingredients, broadly including emissions from Earth's atmosphere, or airglow, Galactic emissions, and extragalactic emissions. The Galactic component has been shown to be dominated by interstellar UV radiation scattered isotropically by dust, but also includes molecular hydrogen fluorescence, H II two-photon emission, and hot gas line emission, in smaller quantities (Murthy 2009; Bowyer 1991). The extragalactic component is dominated by UV flux from resolved sources (i.e., galaxies), but may also include weak emission from the IGM. Measurements of the resolved UV EBL can be determined from catalogs of extragalactic sources and can be interpreted as an average measurement of the star formation rate density over cosmological time, setting a lower limit for the total UV background light. Commonly, measurements of the UV background radiation that do not directly include these resolved sources are termed "diffuse background" measurements. Earlier studies making measurements of the diffuse FUV background are discussed in thorough reviews by Bowyer (1991) and Henry (1991), while more recent work has been reviewed by Murthy (2009). The definition of FUV wavelength coverage for each study varies between 912 and $1740 \AA$, depending on the detector used.

Several techniques have been imparted in order to measure the diffuse FUV background. First, many studies have measured Galactic dust scattering, removing airglow effects, and fitting models to diffuse observations, extrapolating the signal down to zero column density $\left(N_{\mathrm{H}_{\mathrm{I}}}=0\right)$ which provides levels for what is interpreted as the FUV extragalactic background (i.e., galactic sources and potentially diffuse IGM emission). Henry \& Murthy (1993) used this technique to reanalyze data from the Johns Hopkins Ultraviolet Explorer experiment for observations above $|b|=40^{\circ}$ (where $b$ is Galactic latitude). An improved model simulating scattering of diffuse galactic light in the ISM was developed and used by Witt \& Petersohn (1994) to re-measure the extragalactic background in Dynamic Explorer 1 observations from Fix et al. (1989). This same model was used by Witt et al. (1997) to re-evaluate the extragalactic background extrapolation from Far-Ultraviolet Space Telescope observations (Sasseen et al. 1995). Schiminovich et al. (2001) derived the extragalactic FUV background with data from the Narrowband Ultraviolet Imaging Experiment, the first experiment primarily designed to map the FUV background. Most recently, this extrapolation technique has been used by Seon et al. (2010) to measure the FUV extragalactic background with the Spectroscopy of Plasma Evolution from Astrophysical Radiation instrument. A second technique, that measures the truly diffuse extragalactic background, has been imparted by Brown et al. (2000) who masked the resolved FUV sources down to $m_{\mathrm{AB}}=29$ in HST STIS HDF-N and S, and HDF-N parallel imaging (Gardner et al. 2000a). They found a large unresolved diffuse background component that may include contributions from airglow.

Other studies have used FUV spectra and imaging from large data sets to map the FUV background over a large range of Galactic latitudes, revealing patchy skymaps of the background due to variations in intensities of the flux at different latitudes. Murthy et al. (1999) mapped the FUV background over the sky from 17 years of Voyager observations with the Voyager Ultraviolet Spectrometer, unique in that they are not partial to airglow effects, and Murthy \& Sahnow (2004) mapped the FUV background intensity with Far-Ultraviolet Spectroscopic Explorer observations in 71 independent fields. Most recently, Murthy et al. (2010) used archival GALEX imaging to map the diffuse FUV background over $\sim 75 \%$ of the sky. This technique is used to put an upper limit on the extragalactic FUV background from values determined in the darkest areas of these data sets, primarily, but not necessarily, found in the vicinity of the Galactic poles. These FUV background skymaps have also revealed that some of the brightest FUV intensities are correlated with Galactic structures such as molecular clouds and nebulae. Detailed analysis to disentangle components of and effects on the diffuse FUV background in the vicinity of these structures have been carried out by determining correlations with $\mathrm{H}_{\mathrm{I}}$ column, $\mathrm{H}_{2}$ fluorescence, Galactic extinction, and dust scattering, in some cases, resulting in measurements of an FUV extragalactic background component (Sujatha et al. 2005, 2007; Lee et al. 2006). Measurements of the diffuse FUV background are complimented by measurements of the resolved FUV background from extragalactic sources.

In this study, we calculate the FUV EBL from resolved sources in the FUV data used for number counts, and these results are given in Table 2. We use both sets of bright GALEX number counts in our calculation, giving us two possible values for the integrated EBL. First, we fit a slope of $0.13 \pm 0.05$ with an intercept of $0.68 \pm 1.23$ to our FUV number counts for magnitudes 24.5-28.5, including only the faint end of the SBC/ FUV number counts distribution. We also fit a slope of $0.53 \pm$ 0.01 with an intercept of $-9.11 \pm 0.28$ to the XU05 GALEX counts for magnitudes 14.2-23.7. For the HAM10 field GALEX counts, we use the slope of 0.5 fitted to the FUV data by Hammer et al. (2010) with an intercept of $-8.7 \pm 0.81$ for magnitudes 17.25-23.25. Next, these slopes, as well as the number counts, are converted to units of EBL per magnitude bin, erg s${ }^{-1} \mathrm{~cm}^{-2}$ $\mathrm{Hz}^{-1} \mathrm{Sr}^{-1}$, using the formula from Madau \& Pozzetti (2000):

$$
I_{v}=10^{-0.4\left(\mathrm{FUV}_{\mathrm{AB}}+48.6\right)} N\left(\mathrm{FUV}_{\mathrm{AB}}\right) .
$$

Finally, we integrate under each function. For the combined fit of the faint-end SBC/FUV data with the XU05 data (hereafter EBL I), we set $\mathrm{FUV}_{\mathrm{AB}}=24.67$ as the upper limit for the integral of the XU05 function and the lower limit for the integral of the SBC/FUV function, because this 
Table 2

Measurements of the Resolved FUV Background Light

\begin{tabular}{|c|c|c|c|c|c|}
\hline Investigators & Instrument & $\begin{array}{c}\lambda \\
(\AA)\end{array}$ & $\begin{array}{l}\text { Magnitudes Covered } \\
\text { (AB) }\end{array}$ & $\begin{array}{c}\text { FUV BL } \\
\left(\mathrm{nW} \mathrm{m} \mathrm{m}^{-2} \mathrm{sr}^{-1}\right)\end{array}$ & $\begin{array}{c}\text { FUV BL } \\
\text { (photons s} \mathrm{cm}^{-1} \mathrm{sr}^{-1} \AA^{-1} \text { ) }\end{array}$ \\
\hline This work: EBL I ${ }^{\mathrm{a}}$ & $\mathrm{SBC} / G A L E X$ & $1614 / 1530$ & $14.70-29.30$ & $1.3_{-0.2}^{+0.2}$ & $65.9_{-8}^{+8}$ \\
\hline Xu et al. (2005) & GALEX & 1530 & Extrap. to zero mag & $1.03 \pm 0.15$ & $52 \pm 7$ \\
\hline Gardner et al. (2000a) & STIS/FOCA & 1595 & $17.50-29.50$ & $2.9_{-0.4}^{+0.6}$ to $3.9_{-0.8}^{+1.1}$ & $144_{-19}^{+28}$ to $195_{-39}^{+59}$ \\
\hline Armand et al. (1994) & $\ldots \mathrm{c}$ & 2000 & $15.00-18.50$ & $0.8-2.6^{-0.0}$ & $40-130$ \\
\hline
\end{tabular}

Notes.

${ }^{a}$ Bright-end fit is from Xu et al. (2005) GALEX FUV number counts.

$\mathrm{b}$ Bright-end fit is from Hammer et al. (2010) GALEX FUV number counts.

${ }^{\mathrm{c}}$ His measurement is from a prediction of number counts based on galaxy evolution models and published galaxy SEDs.

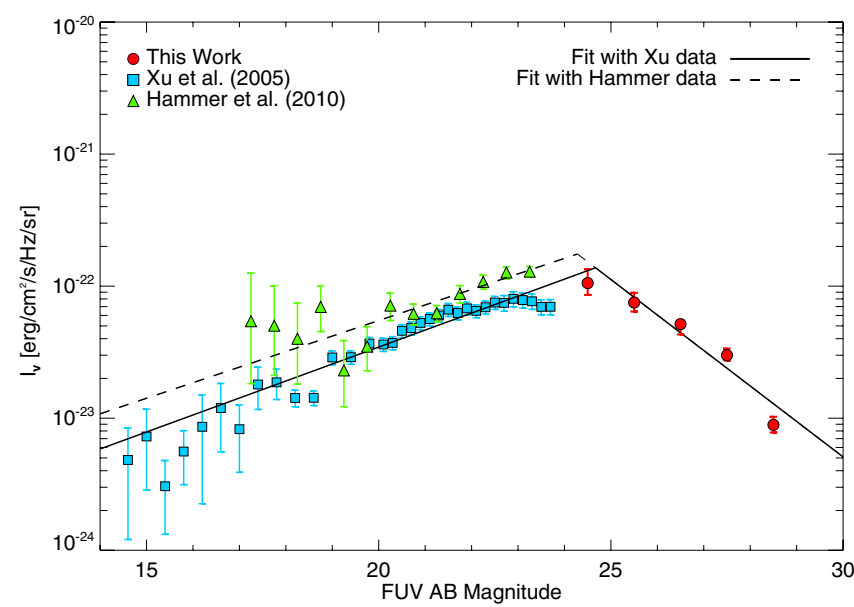

Figure 6. Extragalactic background light from resolved sources per magnitude as a function of FUV magnitude. Two measurements are made from these data. The solid line measures the integrated EBL using the $\mathrm{Xu}$ et al. (2005) counts for the bright end (EBL I), while the dashed line makes this measurement using the Hammer et al. (2010) counts at the bright end (EBL II).

(A color version of this figure is available in the online journal.)

magnitude is the maximum in integrated light. From this model, we measure the integrated EBL for the magnitude range $\mathrm{FUV}_{\mathrm{AB}}=14-30$ of $\nu I_{v}=1.3_{-0.2}^{+0.2} \mathrm{nW} \mathrm{m}^{-2} \mathrm{sr}^{-1}$, or in photon units, $I_{\lambda}=65.9_{-8}^{+8}$ photons $\mathrm{s}^{-1} \mathrm{~cm}^{-2} \mathrm{sr}^{-1} \AA^{-1}$. The errors are $1 \sigma$ uncertainties on the number counts. The models and data are plotted in Figure 6. The model for the GALEX data between $\mathrm{FUV}_{\mathrm{AB}}=14-24.67$ accounts for $66.5 \%$ of EBL I, measuring more of the resolved background light than our faint-end number counts. For the combined fit of the faint-end SBC/FUV data with the HAM10 data (hereafter EBL II), we set $\mathrm{FUV}_{\mathrm{AB}}=24.28$ as the upper limit for the integral of the HAM10 function and the lower limit for the integral of the SBC/FUV function. From this model we measure the integrated EBL for the magnitude range $\mathrm{FUV}_{\mathrm{AB}}=17-30$ of $\nu I_{v}=1.6_{-0.2}^{+0.2} \mathrm{nW} \mathrm{m}^{-2} \mathrm{sr}^{-1}$, or in photon units, $I_{\lambda}=82.6_{-12}^{+12}$ photons s${ }^{-1} \mathrm{~cm}^{-2} \mathrm{sr}^{-1} \AA^{-1}$. Again, the GALEX portion of the model measures more resolved background light than our number counts, accounting for $66 \%$ of EBL II, very similar to XU05. This similarity is due to a caveat in the data included from these two studies in that XU05 cover a larger magnitude range than HAM10, and the latter has higher $I_{\nu}$. This can be clearly seen in Figure 6.

One of the first attempts at determining the FUV background from light emitted by galaxies was carried out by Martin \& Bowyer (1989). They obtained data from an FUV imaging experiment that used a rocket mounted detector to observe signatures of galaxies in the integrated FUV background. The experiment covered wavelengths $1350-1900 \AA$ and determined a $1 \sigma$ upper limit for the summed FUV intensity coming from sources $\sim 50$ photons $\mathrm{s}^{-1} \mathrm{~cm}^{-2} \mathrm{sr}^{-1} \AA^{-1}$, that is $\sim 25 \%-40 \%$ lower than our measurements. The UV EBL was measured at $2000 \AA$ by Milliard et al. (1992) from FOCA number counts and by Armand et al. (1994) from predictions of number counts (Armand \& Milliard 1994). While our measurements are well within the range of 40-130 photons $\mathrm{s}^{-1} \mathrm{~cm}^{-2} \mathrm{sr}^{-1} \AA^{-1}$ predicted by Armand et al. (1994), they are much higher than the 23 photons s${ }^{-1} \mathrm{~cm}^{-2} \mathrm{sr}^{-1} \AA^{-1}$ determined from the FOCA number counts between magnitudes 15.0 and 18.5. Comparing our measurements to those from Gardner et al. (2000a), EBL I and EBL II are $\sim 54 \%-66 \%$ and $~ 43 \%-58 \%$ lower, respectively, than their measurements of $2.9_{-0.4}^{+0.6}-3.9_{-0.8}^{+1.1} \mathrm{nW} \mathrm{m}^{-2} \mathrm{sr}^{-1}$ $\left(144_{-19}^{+28}-195_{-39}^{+59}\right.$ photons $\left.\mathrm{s}^{-1} \mathrm{~cm}^{-2} \mathrm{sr}^{-1} \AA^{-1}\right)$ at $1595 \AA$. Xu et al. (2005) extrapolated models fit to the GALEX FUV number counts $(1530 \AA)$, integrated these functions to zero flux, and measured the total FUV EBL to be $1.03 \pm 0.15 \mathrm{nW} \mathrm{m}^{-2} \mathrm{sr}^{-1}$ which is $\sim 21 \%$ lower than EBL I, $\sim 37 \%$ lower than EBL II, and also below the Gardner et al. (2000a) range. The conclusion that can be drawn from our measurements is that the resolved EBL is unlikely to be much greater than $\sim 100$ photons $\mathrm{s}^{-1} \mathrm{~cm}^{-2} \mathrm{sr}^{-1} \AA^{-1}$, and therefore other diffuse EBL measurements with significantly higher values (Schiminovich et al. 2001; Brown et al. 2000; Witt et al. 1997; Witt \& Petersohn 1994; Henry \& Murthy 1993) almost certainly include Galactic contributions and potentially smaller contributions from airglow. All values for the resolved FUV EBL discussed in this section are summarized in Table 2.

\section{SUMMARY}

We have presented FUV galaxy number counts at $1614 \AA$ measured from deep HST ACS/SBC observations of the HUDF area of the GOODS-S field, the HDF-N area of the GOODS-N field, and 15 smaller fields at various pointings in GOODS-N and $\mathrm{S}$. We sample the faint end of the FUV number counts out to $\mathrm{FUV}_{\mathrm{AB}} \sim 29$, with the majority of the sources in magnitude bins 23.5-28.5, and cover an area $\left(15.9 \operatorname{arcmin}^{2}\right) \sim 4$ times larger than the most recent deep FUV number counts survey (Teplitz et al. 2006) at these wavelength and magnitude ranges. The number counts distribution provides the following results.

1. A slope of $0.13 \pm 0.04$ (intercept of $0.68 \pm 1.23$ ) fits the faint end of the logarithmic number counts distribution from $\mathrm{FUV}_{\mathrm{AB}}=24.5$ to 28.5 . 
2. These number counts are $\sim 35 \%$ and $\sim 36 \%$ lower, on average, than the faint FUV counts measured in the HDF-N area of GOODS-N from Gardner et al. (2000a) and Teplitz et al. (2006), respectively. The differences are most likely due to cosmic variance.

3. The bright end of the number counts slope, at $\mathrm{FUV}_{\mathrm{AB}}=$ 22.5 , intersects the most recent GALEX FUV number counts from Hammer et al. (2010), but is higher than the GALEX FUV counts from Xu et al. (2005) at all common magnitudes.

4. The latest $\lambda$ CDM SAM based on the WMAP5 cosmology (Somerville et al. 2011) is in good agreement with the FUV number counts. Generally, the FUV counts are higher than the SB4/Ly $\alpha$-flat single SED model (Xu et al. 2005) but become more consistent at the faint end. This may result from the model being based on a single starburst SED, thus offering evidence for number density evolution.

5. The integrated light from field galaxies contributes $1.3_{-0.2}^{+0.2} \mathrm{nW} \mathrm{m}^{-2} \mathrm{sr}^{-1}$ or $65.9_{-8}^{+8}$ photons s $\mathrm{sm}^{-1} \mathrm{sr}^{-1} \AA^{-1}$ to the FUV EBL for magnitudes 14-30 when measured with XU05 bright-end GALEX counts, and $1.6_{-0.2}^{+0.2} \mathrm{nW} \mathrm{m}^{-2} \mathrm{sr}^{-1}$ or $82.6_{-12}^{+12}$ photons $\mathrm{s}^{-1} \mathrm{~cm}^{-2} \mathrm{sr}^{-1} \AA^{-1}$ for magnitudes 17-30 when measured with HAM10 bright-end GALEX counts. The GALEX portion of these models accounts for $\sim 66 \%$ of the total integrated light in each case. This measurement sets a lower limit for future calculations of the diffuse background. The resolved EBL is unlikely to be much greater than $\sim 100$ photons $\mathrm{s}^{-1} \mathrm{~cm}^{-2} \mathrm{sr}^{-1} \AA^{-1}$. Any measurement that yields values significantly higher than this value almost certainly includes Galactic and airglow contributions.

We are grateful to the anonymous referee for their helpful comments that improved this paper. We thank R. S. Somerville and R. C. Gilmore for providing us with their semi-analytic model and for numerous helpful discussions and comments on this paper. We also thank D. Hammer for useful science discussions and sharing number count results, and $\mathrm{C}$. K. Xu for providing us with theoretical number count models. Support for Program numbers GO-10403 and GO-10872 was provided by NASA through a grant from the Space Telescope Science Institute, which is operated by the Association of Universities for Research in Astronomy, Incorporated, under NASA contract NAS5-26555. E.N.V. was funded by the NASA Graduate Student Research Program grant no. NNX08AR95H.

This research has made use of data obtained from the Chandra Source Catalog, provided by the Chandra X-ray Center (CXC) as part of the Chandra Data Archive.

This research has made use of the NASA/IPAC Infrared Science Archive, which is operated by the Jet Propulsion Laboratory, California Institute of Technology, under contract with the National Aeronautics and Space Administration.

\section{REFERENCES}

Armand, C., \& Milliard, B. 1994, A\&A, 282, 1

Armand, C., Milliard, B., \& Deharveng, J. M. 1994, A\&A, 284, 12
Arnouts, S., et al. 2005, ApJ, 619, L43

Beckwith, S. V. W., et al. 2006, AJ, 132, 1729

Bertin, E., \& Arnouts, S. 1996, A\&AS, 117, 393

Bowyer, S. 1991, ARA\&A, 29, 59

Bridge, C. R., et al. 2010, ApJ, 720, 465

Brown, T. M., Kimble, R. A., Ferguson, H. C., Gardner, J. P., Collins, N. R., \& Hill, R. S. 2000, AJ, 120, 1153

Bruzual, G., \& Charlot, S. 2003, MNRAS, 344, 1000

Cardelli, J. A., Clayton, G. C., \& Mathis, J. S. 1989, ApJ, 345, 245

Charlot, S., \& Fall, S. M. 2000, ApJ, 539, 718

Cohen, J. G., Hogg, D. W., Blandford, R., Cowie, L. L., Hu, E., Songaila, A., Shopbell, P., \& Richberg, K. 2000, ApJ, 538, 29

Cowie, L. L., Barger, A. J., \& Trouille, L. 2009, ApJ, 692, 1476

Dahlen, T., et al. 2010, ApJ, 724, 425

Deharveng, J.-M., Sasseen, T. P., Buat, V., Bowyer, S., Lampton, M., \& Wu, X 1994, A\&A, 289, 715

Evans, I. N., et al. 2010, ApJS, 189, 37

Fix, J. D., Craven, J. D., \& Frank, L. A. 1989, ApJ, 345, 203

Gardner, J. P., Brown, T. M., \& Ferguson, H. C. 2000a, ApJ, 542, L79

Gardner, J. P., et al. 2000b, AJ, 119, 486

Gehrels, N. 1986, ApJ, 303, 336

Giavalisco, M., et al. 2004, ApJ, 600, L93

Gilmore, R. C., Madau, P., Primack, J. R., Somerville, R. S., \& Haardt, F. 2009, MNRAS, 399, 1694

Hammer, D., et al. 2010, ApJS, 191, 143

Henry, R. C. 1991, ARA\&A, 29, 89

Henry, R. C., \& Murthy, J. 1993, ApJ, 418, L17

Hoversten, E. A., et al. 2009, ApJ, 705, 1462

Iglesias-Páramo, J., Buat, V., Donas, J., Boselli, A., \& Milliard, B. 2004, A\&A, 419, 109

Kinney, A. L., Calzetti, D., Bohlin, R. C., McQuade, K., Storchi-Bergmann, T., \& Schmitt, H. R. 1996, ApJ, 467, 38

Lee, D.-H., et al. 2006, ApJ, 644, L181

Madau, P., \& Pozzetti, L. 2000, MNRAS, 312, L9

Martin, C., \& Bowyer, S. 1989, ApJ, 338, 677

Milliard, B., Donas, J., Laget, M., Armand, C., \& Vuillemin, A. 1992, A\&A, 257,24

Murthy, J. 2009, Ap\&SS, 320, 21

Murthy, J., Hall, D., Earl, M., Henry, R. C., \& Holberg, J. B. 1999, ApJ, 522, 904

Murthy, J., Henry, R. C., \& Sujatha, N. V. 2010, ApJ, 724, 1389

Murthy, J., \& Sahnow, D. J. 2004, ApJ, 615, 315

Pimbblet, K. A., Drinkwater, M. J., \& Hawkrigg, M. C. 2004, MNRAS, 354, L61

Sasseen, T. P., Lampton, M., Bowyer, S., \& Wu, X. 1995, ApJ, 447, 630

Schiminovich, D., Friedman, P. G., Martin, C., \& Morrissey, P. F. 2001, ApJ, 563, L161

Schlegel, D. J., Finkbeiner, D. P., \& Davis, M. 1998, ApJ, 500, 525

Seon, K.-I., et al. 2010, arXiv:1006.4419

Siana, B., et al. 2007, ApJ, 668, 62

Siana, B., et al. 2010, ApJ, 723, 241

Somerville, R. S., Gilmore, R. C., Primack, J. R., \& Dominguez, A. 2011, arXiv: 1104.0669

Somerville, R. S., Hopkins, P. F., Cox, T. J., Robertson, B. E., \& Hernquist, L. 2008, MNRAS, 391, 481

Somerville, R. S., Lee, K., Ferguson, H. C., Gardner, J. P., Moustakas, L. A., \& Giavalisco, M. 2004, ApJ, 600, L171

Somerville, R. S., \& Primack, J. R. 1999, MNRAS, 310, 1087

Somerville, R. S., Primack, J. R., \& Faber, S. M. 2001, MNRAS, 320, 504

Sujatha, N. V., Murthy, J., Shalima, P., \& Henry, R. C. 2007, ApJ, 665, 363

Sujatha, N. V., Shalima, P., Murthy, J., \& Henry, R. C. 2005, ApJ, 633, 257

Teplitz, H. I., et al. 2006, AJ, 132, 853

Williams, R. E., et al. 1996, AJ, 112, 1335

Witt, A. N., Friedmann, B. C., \& Sasseen, T. P. 1997, ApJ, 481, 809

Witt, A. N., \& Petersohn, J. K. 1994, in ASP Conf. Ser. 58, The First Symposium on the Infrared Cirrus and Diffuse Interstellar Clouds, ed. R. M. Cutri \& W. B. Latter (San Francisco, CA: ASP), 91

Wyder, T. K., et al. 2005, ApJ, 619, L15

Xu, C. K., et al. 2005, ApJ, 619, L11 